\title{
El uso de pero enfático en el corpus PRESEEA de Santiago de Chile
}

\author{
The use of emphatic pero in the PRESEEA corpus from Santiago de Chile \\ Silvana Arriagada Anabalón ${ }^{1}$ y Silvana Guerrero González ${ }^{2}$ \\ ${ }^{1}$ Pontificia Universidad Católica de Chile. Correo electrónico: sbarriagada@uc.cl \\ ${ }^{2}$ Universidad de Chile. Correo electrónico: siguerrero@u.uchile.cl
}

\begin{abstract}
En esta investigación se analiza el comportamiento del pero enfático a nivel discursivo y su estratificación sociolingüística en el corpus del Proyecto Para el Estudio Sociolingüístico del Español de España y América (PRESEEA) correspondiente al habla de Santiago de Chile. Primero se determina cuáles son los elementos enfatizados con pero. Luego, se establece la frecuencia de empleo de dicha partícula, para comprobar si existe variación entre su uso y los factores sociodemográficos de los hablantes. Además, se correlaciona su frecuencia de empleo con el tipo de secuencia discursiva (narrativa o argumentativa) en la que se registra. El análisis de los datos nos permitió concluir que los elementos enfatizados con pero son sustantivos, adjetivos, adverbios, reduplicaciones y otros, como oraciones. El análisis demuestra que el elemento en estudio es mayormente empleado en discursos narrativos de mujeres y que se trata de un recurso más propio del habla joven de la comunidad de habla en estudio.
\end{abstract}

Palabras clave: sociolingüística variacionista, variación discursiva, oralidad, pero enfático

This investigation is concerned with the use of pero at a discourse level and its sociolinguistic stratification in the corpus of the Proyecto Para el Estudio Sociolingüístico del Español de España y América (PRESEEA), corresponding to Spanish native speakers from Santiago de Chile. Firstly, the study determines the elements that are emphasized through the use of pero. Secondly, the frequency of the particle's use is established in order to determine any variation between its utilization and the speakers' sociodemographic factors. In addition, the analysis seeks to correlate the frequency of use with the type of discursive sequence (narrative or argumentative) in which it is found. Data analysis allowed us to conclude that the elements emphasized through pero are nouns, adjectives, adverbs, reduplications and others, such as sentences. The study demonstrates that this particle is mainly employed in the narrative discourse of women, and that it is a resource mostly found in youth language from the speech community under investigation.

Key words: variationist sociolinguistics, discursive variation, orality, emphatic pero 


\section{INTRODUCCIÓN}

\subsection{Naturaleza, alcance y objetivos del estudio}

La partícula pero en la lengua española no sólo permite dar cuenta de una adversación entre dos oraciones o entre miembros oracionales, sino que, en muchas ocasiones, presenta un uso enfático donde funciona como un indicador de modalidad (Acín 1993-1994). Por ejemplo, en "lamentablemente el vocabulario deja mucho que desear pero mucho mucho" (SCHI_H31_025) ${ }^{3}$, observamos que el informante emplea la partícula pero para reforzar y resaltar la idea que venía desarrollando. Dicha partícula podría elidirse fácilmente, sin embargo, el enunciado perdería, en parte, la función enfática.

En este trabajo se realiza una aproximación sociolingüística, en el marco del variacionismo, al empleo del pero enfático en una muestra de 108 narraciones de experiencia personal y 108 discursos argumentativos, extraídos del Corpus del Proyecto para el Estudio Sociolingüístico del Español de España y América (PRESEEA) correspondiente al habla de Santiago de Chile. El objetivo general de este estudio es analizar el comportamiento del pero enfático a nivel discursivo y su estratificación sociolingüística en el mencionado corpus. Nuestros objetivos específicos son, primero, determinar cuáles son los elementos -sustantivos, adjetivos, adverbios, reduplicaciones u otros- enfatizados con pero. Luego, establecer la frecuencia de empleo de dicha partícula. En tercer lugar, comprobar si existe variación entre los factores sociodemográficos de los hablantes -sexo, edad y nivel educacional- y el empleo del elemento variable analizado y, finalmente, correlacionar su frecuencia de empleo con el tipo de secuencia discursiva (narrativa o argumentativa) en la que se registra. La hipótesis que guía nuestro trabajo es que las características sociodemográficas de los hablantes de español en Santiago de Chile están correlacionadas con el empleo del pero enfático utilizado en diferentes secuencias discursivas. En concreto, la hipótesis intra-grupal es que, en todos los casos, son las mujeres las que utilizan más esta partícula con función enfática, mientras que la hipótesis inter-grupal supone que el empleo de este recurso se da mayoritariamente en mujeres jóvenes con nivel educacional alto.

En este contexto, destacamos que los estudios que han abordado el funcionamiento del pero con valor enfático, lo han hecho más bien desde el punto de vista descriptivo, dando cuenta de cómo funciona en términos gramaticales y lexicográficos, pero no se han propuesto analizar su comportamiento en secuencias discursivas diferentes y menos desde el punto de vista sociolingüístico. Por lo anterior, este trabajo no sólo contribuye a la descripción teórica de la mencionada partícula, sino que se perfila como una contribución a la descripción sociolingüística y pragmática del español hablado en Santiago de Chile.

Los ejemplos extraídos del corpus están codificados de la siguiente manera: $\mathrm{SCHI}=$ Santiago de Chile; $\mathrm{H}=$ Hombre y $\mathrm{M}=$ Mujer. El primer número, luego del sexo del informante, corresponde al grupo etario: 1=20-34 años; 2= 35-54 años y $3=55$ años y más. El segundo número corresponde al nivel educacional del informante: $1=$ estudios básicos completos o incompletos; $2=$ estudios medios completos o incompletos y $3=$ estudios universitarios completos o incompletos. El número que sigue al guión corresponde al número correlativo que reciben los sujetos que conforman la muestra. Los ejemplos están transcritos en ortografía convencional y los fragmentos de discurso referido se ponen entre comillas. El empleo de pero enfático se destaca con cursiva. 


\section{MARCo CONCEPTUAL}

\subsection{El pero enfático y su aplicación en estudios de variación sociolingüística}

En el marco del variacionismo laboviano (Labov 1983 [1972]), en esta investigación nos proponemos analizar el empleo del pero enfático en una muestra de discurso natural en hablantes santiaguinos, a fin de contribuir al estudio del registro coloquial de la lengua española. Como sabemos, la variación puede manifestarse en los distintos niveles de la lengua, esto es, fonético-fonológico, gramatical (morfológico y sintáctico), léxico y pragmático-discursivo. Moreno Fernández (2012) sostiene que el uso lingüístico se convierte en un concepto clave para explicar la variación, ya que, por una parte, determina y refleja la variabilidad interna y, por otra, es sensible a la diversidad de condicionamientos externos, adecuándose a ella. Sin embargo, la variación pragmático-discursiva ha sido una de las menos tratadas en los estudios sociolingüísticos, por lo que es necesario comenzar a incorporar varios factores a la descripción lingüística, en lo fundamental, lo pragmático, lo social, lo cognitivo, lo comunicativo y lo contextual (Serrano 2011). En este estudio, el eje fundamental es el vínculo entre lo discursivo-pragmático y lo social, pues el fenómeno que nos atañe ejerce una función trascendental en el discurso, porque podría describirse desde su función polifónica, puesto que al emplearse está presuponiendo que el interlocutor no hizo la inferencia adecuada. A este respecto, van Dijk pone de relieve que:

De esta situación social y de su conocimiento del hablante (que puede ser nulo, aunque en la interacción cara a cara el oyente infiere mucho sobre el hablante de la comunicación paratextual), el oyente puede inferir los verdaderos conocimientos, deseos y evaluaciones del hablante para poder asignar el acto de habla correcto. Esta información social y cognoscitiva será combinada para formar esquemas pragmáticos, que son medios estratégicos para decidir cómo cabe la emisión en el esquema (van Dijk 1996: 96).

Junto con lo anterior, en esta investigación partimos de la base de que gran parte de los hechos de variación lingüística varían en forma sistemática, si se los correlaciona con variables sociodemográficas como el grupo socioeconómico ${ }^{3}$, la edad y el sexo de los informantes. Esto implica que el hecho de que las variables lingüísticas estén en covariación sistemática con las variables externas permite a los individuos instalarse en un determinado espacio del espectro social. En relación con esto, Coates pone de relieve que "aunque desde el punto de vista lingüístico las variables lingüísticas sean formas equivalentes para referirse a algo en particular, son diferentes desde una perspectiva social" (Coates 2009: 89). Por su parte, Moreno Fernández (1998) y Blas Arroyo (2005) también señalan que no es posible reconocer de antemano qué tipo de variables sociales van a actuar sobre la variación lingüística, primero, porque la variabilidad no tiene por qué manifestarse de la misma forma en comunidades de habla diferentes $y$, segundo, porque estos mismos factores no se configuran de la misma forma en sociedades distintas. Como ya hemos señalado, los factores sexo, edad y nivel educacional serán las variables independientes empleadas en esta

En esta investigación trabajaremos solo con la variable nivel educacional, pues la muestra empleada no utiliza un sistema de post-estratificación (cf. 3.1.1.). 
investigación sobre el carácter que asume la variación lingüística correspondiente al empleo del pero enfático en una muestra del español hablado en Santiago de Chile.

\subsection{Caracterización del pero enfático}

La gramática tradicional ha considerado el pero como una conjunción adversativa que expresa contraposición: “con la conjunción pero se contraponen dos ideas. Normalmente, una de ellas se expresa en el término de dicha conjunción, mientras que la otra, opuesta a la primer, se infiere del primer segmento coordinado" (RAE, 2009: 2451). Sin embargo, no se establece una contraposición absoluta entre dos segmentos, sino más bien se relacionan con lo que la RAE denominará inferencias y supone la perspectiva del hablante. En el enunciado "no sé si está bien el ejemplo que te voy a dar pero yo no comprendo que haya gente rica/ por ejemplo hay personas ricas que llegan a comer por hobbie" (SCHI_H11_005), no se contrapone la comprensión del hablante con su habilidad de encontrar un buen ejemplo, sino más bien ambas ideas se oponen de acuerdo a la perspectiva del hablante y lo que para él es relevante en determinada situación. Así, "las inferencias que determinan la interpretación de la conjunción pero son de deducciones libres establecidas en función de muy diversas informaciones" (RAE, 2009: 2453).

En la misma línea anterior, se reconoce la forma pero si como una réplica enfática en la que de alguna manera se rebate a través de un énfasis una afirmación previa que el hablante consideraría importante, por ejemplo, en el siguiente uso de pero si se enfatiza un reparo del hablante a lo dicho por su padre: "sí/ mi papá dice 'yo no sé por qué se quejan tanto del Transantiago' y yo le digo 'pero si tú no lo usái cómo podís decir eso"'. En este caso, la partícula pero si se usa como un conector discursivo que muestra una reacción del hablante ante lo dicho o sucedido.

Gili Gaya (1961) señala que, en algunas oportunidades, las conjunciones pueden expresar transiciones o conexiones mentales que trascienden el sentido oracional. Es aquí donde se ubicaría la partícula pero, que puede ir al comienzo de una cláusula para anunciar algún tipo de restricción al sentido general de lo que ya se ha dicho:

\footnotetext{
En esta posición tiene a veces uso enfático destinado a manifestar sorpresa, extrañeza, asombro o a interrumpir en la conversación con una frase ajena a la misma. A menudo se acentúa en la pronunciación: Pero ¿cómo lo has sabido?; Pero iqué horror!; Pero fijate en ese que viene. Con este valor enfático la usamos también dentro de las frases exclamativas, en las cuales pierde todo valor adversativo: ;Bien!, ipero que muy bien! (Gili Gaya, 1961: 282).
}

En este mismo orden, Alarcos Llorach (1999), dentro de las conjunciones adversativas, sitúa a la partícula pero. No obstante, también señala que dicha partícula puede encabezar una secuencia sin conexión directa con algo precedente, adoptando, en consecuencia, un valor enfático que le otorga función adverbial. A este tipo de uso es al que denominamos pero enfático, ya que adquiere un sentido aumentativo (Acín 1993-1994). En este contexto, también el DRAE (2001), en su segunda acepción, señala que se trata de una conjunción adversativa usada "a principio de cláusula sin referirse a otra anterior, para dar énfasis o fuerza de expresión a lo que se dice. Pero ¿dónde vas a meter tantos libros? Pero iqué hermosa noche!" (s.v. pero). Asimismo, bajo esta voz alude a la expresión "pero que muy" y propone que 
es una expresión usada "ante adjetivos y adverbios para darles mayor relieve. Toca el clarinete pero que muy bien". En este contexto, también van Dijk (1996: 65 y 68) señala que en un caso como “A: Está bien, vámonos. B: ¡Pero aún no estoy listo!”, el pero expresado por $\mathrm{B}$ indica que el segundo hablante no acepta, o incluso protesta, el acto de habla del primer hablante.

A diferencia de la partícula pero con función coordinativa adversativa, el pero enfático, al no enlazar miembros equifuncionales, puede eliminarse de la construcción y sólo genera una pérdida o una aminoración del énfasis (Acín, 1993-1994), como ocurre en el siguiente ejemplo, extraído de una narración de la muestra analizada: "se suponía que era casi la misma hora que tenía que llegar a la casa /entonces fue como todo como que / concordó todo entonces fue pero atroz" (SCHI_M21_020). Junto con este antecedente que responde a una prueba sintáctica, Nicolás Cantabella y Hernández Rubio (2011) sintetizan cuatro características esenciales de la partícula en estudio, a saber, 1) sólo realza y puede ser eliminada de la construcción, lo que conlleva sólo un aminoramiento del énfasis; 2) no pierde el valor contrapositivo, pues es implícito; 3) el elemento introducido por esta partícula manifiesta sorpresa, que supone contraposición con lo normalmente esperable; $y, 4)$ presenta entonación ascendente, según el grado de énfasis, y una pausa que puede estar marcada gráficamente o no.

De acuerdo con lo anteriormente expuesto, tres serían los tipos de pero enfático: a) el que se usa para expresar sorpresa por parte del hablante, b) el que se usa para manifestar desacuerdo por parte del hablante y c) el que se usa para mostrar impaciencia y enfado por parte del hablante. En este estudio hemos encontrado un cuarto sentido para la partícula en estudio, a saber, el de necesidad o requerimiento de información, por ejemplo: “pero ¿qué pasó?”, donde el interlocutor supone que el otro dará más información.

En otro orden, dentro de los elementos que han sido descritos como enfatizados con pero, están los sustantivos, los adjetivos, los adverbios y las reduplicaciones. Para cumplir los objetivos de esta investigación, sintetizamos sus principales usos y características en el Cuadro 1.

En esta investigación, hemos incluido también, entre los elementos enfatizados, las oraciones completas, entendidos estos casos como aquellos en los que el pero intensifica el sentido de una construcción oracional, y no una parte de ella. Un ejemplo de esto, obtenido del corpus, es el siguiente: "[sobre el Transantiago] eeh el que hace es que muy lento / hay poco / quizás/ por/ sería / porque estábamos tan acostumbrados a las amarillas / pero / las amarillas andaban unas tras otro y / y soplados" (SCHI_H31_030). En este caso, semánticamente se refuerza y enfatiza la rapidez del transporte aludido. Este significado se expresa a través de una oración que es enfatizada por el pero, es decir, no se están contraponiendo las ideas, sino que se están reforzando características positivas (frecuencia y rapidez) a partir de la costumbre al antiguo sistema de transporte en comparación al sistema actual. En concreto, el pero sólo realza el sentido oracional y puede ser eliminado de la construcción, lo que conlleva sólo un aminoramiento del énfasis y, además, no se pierde el valor contrapositivo, de acuerdo con Nicolás Cantabella y Hernández Rubio (2011).

En suma, el pero enfático se asocia a un sentido aumentativo (de énfasis) que recae sobre distintos elementos del discurso. En este sentido, se constituye como uno de los recursos con los que se cuenta para enfatizar, realzar o remarcar una idea. 
Este sentido estaría asociado, según nuestra propuesta, a expresiones de sorpresa, desacuerdo, enfado e impaciencia. Por lo tanto, se vincularía además con la afectividad y subjetividad del hablante, en cuanto se presenta como un índice apreciativo.

Cuadro 1. Cuadro de elementos enfatizados con pero (Adaptado de Acín, 1993-1994: 226-230 y del Manual de la Nueva gramática de la lengua española, 2010: 616-617)

\begin{tabular}{|c|c|c|c|c|}
\hline \multicolumn{2}{|c|}{ Sustantivos } & \multirow[t]{2}{*}{ Adjetivos } & \multirow[t]{2}{*}{ Adjetivos } & \multirow[t]{2}{*}{ Reduplicaciones } \\
\hline Complemento directo & $\begin{array}{l}\text { Complemento } \\
\text { circunstancial }\end{array}$ & & & \\
\hline $\begin{array}{l}\text { A p a r e c e } n \\
\text { acompañados de un } \\
\text { intensificador, como } \\
\text { mucho, bastante } \\
\text { o demasiado, o de } \\
\text { un sustantivo 'de } \\
\text { cantidad', } \\
\text { cantidad }\end{array}$ & $\begin{array}{l}\text { Expresan modo o } \\
\text { cantidad }\end{array}$ & $\begin{array}{l}\mathrm{R} \text { e } \mathrm{s} \mathrm{p} \text { o } \mathrm{n} \mathrm{d} \text { e } \mathrm{n}, \\
\text { generalmente, a la } \\
\text { combinación pero }+ \\
\text { adjetivo. También } \\
\text { pueden construirse } \\
\text { con "que", a menos } \\
\text { que el adjetivo tenga } \\
\text { forma superlativa - } \\
\text { ísimo/a }\end{array}$ & $\begin{array}{l}\text { E } x \text { p r e s a } n \\
\text { modo, terminados } \\
\text { los tomo } \\
\text { en -mente o en } \\
\text {-ísimo. Incluye } \\
\text { otros adverbios y } \\
\text { puede incluir la } \\
\text { construcción pero } \\
+ \text { gerundio }\end{array}$ & $\begin{array}{l}\text { Une los miembros } \\
\text { realzados. Los } \\
\text { e } 1 \text { e } \mathrm{m} \mathrm{e} \mathrm{n} \mathrm{t} \text { o s } \\
\text { redup } 1 \text { i c a d o s } \\
\text { pueden ser } \\
\text { s u s a n t i v o s, } \\
\text { adjetivos } \\
\text { adverbios. }\end{array}$ \\
\hline $\begin{array}{l}\text { "Comí, pero } \\
\text { muchísimas cerezas"3 }\end{array}$ & $\begin{array}{l}\text { "Baja, pero } \\
\text { relámpago, a la } \\
\text { bodega", donde } \\
\text { "pero relámpago" } \\
\text { equivale } \\
\text { "rápido". }\end{array}$ & $\begin{array}{l}\text { "Tenían un capricho } \\
\text { pero grande con el } \\
\text { dichoso monasterio" }\end{array}$ & $\begin{array}{l}\text { "Escucha lo que } \\
\text { voy a decirte, pero } \\
\text { at en t a m e nte"; } \\
\text { "Vete, pero lejos"; } \\
\text { "Vete, pero } \\
\text { lejísimos"; "Me } \\
\text { voy, pero volando", }\end{array}$ & $\begin{array}{l}\text { "Hace frío, pero } \\
\text { frío" }\end{array}$ \\
\hline
\end{tabular}

\section{Metodología}

\subsection{Corpus}

El corpus que sirvió de base para el análisis del presente estudio consistió en 216 secuencias discursivas, 108 narraciones de experiencia personal y 108 discursos argumentativos, extraídos de la totalidad de entrevistas sociolingüísticas que conforman el corpus de Santiago de Chile en el Proyecto para el Estudio Sociolingüístico del Español de España y América (PRESEEA) ${ }^{3}$. Para la recopilación del corpus sociolingüístico de PRESEEA en Santiago de Chile, se aprovechó la instancia de aplicación de un instrumento que se realiza en el marco de la asignatura de Sociolingüística que se imparte en las Licenciaturas en Lengua y Literatura Hispánica y Lengua y Literatura Inglesas de la Universidad de Chile. Las entrevistas fueron hechas, entre 2007 y 2012, a hombres y mujeres con características sociodemográficas congruentes, aplicando los supuestos metodológicos sugeridos por Labov (1983 [1972]). Consecuentemente, en dichas situaciones los entrevistadores debían tratar

Los ejemplos de esta Tabla son de los autores.

$4 \quad$ El PRESEEA es un proyecto para la creación de un corpus de lengua española hablada representativo del mundo hispánico en su variedad geográfica y social. Esos materiales se reúnen atendiendo a la diversidad sociolingüística de las comunidades de habla hispanohablantes. En la actualidad, el proyecto agrupa a cerca de 40 equipos de investigación sociolingüística. El equipo de PRESEEA en Santiago de Chile está compuesto por los profesores de la Universidad de Chile, Alfredo Matus Olivier (coordinador) y Abelardo San Martín Núñez y Silvana Guerrero González (investigadores). 
de superar la "paradoja del observador"3 consiguiendo, de esta forma, una muestra significativa de discurso natural grabado (vernáculo ${ }^{4}$ ) de hablantes de la comunidad de habla en estudio.

El cuestionario utilizado para la realización de las mencionadas entrevistas consta de varias secciones, entre ellas, las que permiten elicitar las secuencias discursivas que conforman el corpus de nuestra investigación. En relación con esto, es necesario precisar que se seleccionó la narración y la argumentación mejor desarrolladas por cada informante. De esta forma, se puede medir el mejor desempeño de cada uno de ellos y, posteriormente, compararlos. Este criterio de selección de las secuencias mejor desarrolladas nos permitió controlar algunas variables intervinientes, como el cansancio y el desinterés de los sujetos considerando la duración de la entrevista, entre otras que pudieran surgir en el marco de la misma.

\subsubsection{Población de la muestra}

Para la conformación de la muestra de sujetos entrevistados se consideró la población santiaguina ${ }^{5}$. El cuestionario de la entrevista se aplicó a una muestra de sujetos del tipo denominado "muestra por cuotas con afijación uniforme" (López Morales 1994). La muestra así conformada comprende un total de 108 individuos, distribuidos proporcionalmente como se indica en la Tabla 1, que se incluye a continuación.

Tabla 1: Distribución de sujetos de la muestra por nivel educacional, sexo y edad, según PRESEEA

\begin{tabular}{|c|c|c|c|c|c|c|c|}
\hline \multicolumn{10}{|c|}{ Grupo de edad } \\
\hline \multirow{2}{*}{$\begin{array}{c}\text { N i v e 1 } \\
\text { educacional }\end{array}$} & \multicolumn{2}{|c|}{$20-34$} & \multicolumn{2}{|c|}{$35-54$} & \multicolumn{2}{|c|}{55 y más } & \multirow{2}{*}{ Totales } \\
\cline { 2 - 7 } & $\mathrm{H}$ & $\mathrm{M}$ & $\mathrm{H}$ & $\mathrm{M}$ & $\mathrm{H}$ & $\mathrm{M}$ & \\
\hline Alto & 6 & 6 & 6 & 6 & 6 & 6 & 36 \\
\hline Medio & 6 & 6 & 6 & 6 & 6 & 6 & 36 \\
\hline Bajo & 6 & 6 & 6 & 6 & 6 & 6 & 36 \\
\hline Totales & 18 & 18 & 18 & 18 & 18 & 18 & 108 \\
\hline
\end{tabular}

Según Labov (1983 [1972]), el objetivo de la investigación lingüística de una comunidad de habla consiste en estudiar la forma en cómo habla la gente cuando no está siendo sistemáticamente observada; sin embargo, sólo podemos obtener tales datos mediante la observación sistemática. A esto es a lo que se le ha denominado paradoja del observador. Este dilema se soluciona encontrando la manera de completar la entrevista formal con otros datos, o cambiar la estructura de la situación de la entrevista de una u otra manera. En este sentido, una forma de superar esta paradoja consiste en romper las constricciones de la situación de entrevista mediante diversos procedimientos que distraen la atención del sujeto en su propio discurso y pueden hacer brotar su habla más vernácula.

4 Citando a Labov, Moreno Fernández (2012: 181-182) señala que: "El vernáculo, en el que se presta al discurso propio una atención mínima, aporta los datos más sistemáticos por el análisis lingüístico. El vernáculo se define como la forma de hablar adquirida durante la preadolescencia".

5 Por lo que refiere a los criterios de asignación de hablante nativo de Santiago de Chile, de acuerdo con Prieto (19951996: 399), se aplicaron las siguientes restricciones en la selección de los sujetos: 1) haber nacido y residido en forma ininterrumpida en Santiago; 2) haber residido en forma ininterrumpida en Santiago desde los cinco años de edad y 3) haber nacido en Santiago y haber residido en Santiago la mayor parte de sus vidas, salvo por períodos que sumados no superen los cuatro años en el tramo de 55 años y más, y los tres años en el tramo de 35 a 54 años. 


\subsubsection{Procedimiento de estratificación empleado}

Siguiendo el sistema de estratificación del Proyecto PRESEEA, la muestra se estratificó de acuerdo con sus respectivos niveles de instrucción, es decir, según la equivalencia entre los niveles de estudios básico, secundario y superior con los niveles sociales bajo, medio y alto, respectivamente. Sin embargo, con el propósito de garantizar la homogeneidad en la composición de los distintos estratos de la muestra $\mathrm{y}$, por lo tanto, la representatividad de los mismos en relación con la población analizada, se complementó dicho sistema de estratificación con el procedimiento de estratificación empleado por el proyecto de Estudio Sociolingüístico del Español de Chile $(\mathrm{ESECH})^{3}$. Dicho sistema de estratificación considera una escala de estatus socioeconómico que contempla las siguientes variables independientes: nivel educacional, categoría ocupacional y comuna de residencia (San Martín y Guerrero 2015). De este modo, se escogieron las entrevistas realizadas a aquellos sujetos que, tras la aplicación de la escala de estratificación social, mostraban lo que Lenski (1954) ha denominado cristalización o congruencia de estatus ${ }^{4}$.

\section{2. Procedimiento analítico}

De acuerdo con los objetivos trazados en esta investigación, el análisis tuvo, en lo primordial cinco etapas: 1) selección de todos los contextos en que el pero tenía función enfática, 2) determinación de los elementos -sustantivos, adjetivos, adverbios, reduplicaciones u otros- que eran enfatizados, 3) determinación de la frecuencia de empleo en cada caso, 4) correlación entre la partícula en estudio y los factores sociodemográficos de los hablantes -sexo, edad y nivel educacional- y 5) correlación entre la partícula en estudio y el tipo de secuencia discursiva (narrativa o argumentativa) en la que se registra. De este modo, pudimos cumplir nuestro objetivo general, esto es, analizar el comportamiento del pero enfático a nivel discursivo y la estratificación sociolingüística del empleo de dicho mecanismo en el mencionado corpus.

En lo que respecta a la descripción de los procedimientos estadísticos aplicados, en esta investigación incluiremos tanto el análisis descriptivo (frecuencias absolutas y los porcentajes respectivos) como el inferencial (comparación de medias y ji cuadrado). En este último caso, el paquete estadístico empleado es el SPSS (Statistical Package for the Social Sciences), versión 15.0. El grado de significación se definirá en el $5 \%$, según el cual $p<0,05$ será estadísticamente significativo. Dichos procedimientos siguen las sugerencias de Moreno Fernández (1990), López Morales (1994) y Hernández Campoy y Almeida (2005).

ESECH es un grupo de investigación de la Universidad de Chile al que pertenecen los miembros del Equipo PRESEEA-Santiago.

4 Según Lenski (1954), se considera que un individuo es congruente con su estatus cuando las puntuaciones obtenidas en las diferentes dimensiones usadas para medir el estatus, son más o menos iguales, independiente de que sus rangos sean altos, bajos o estén en una extensión media. Cuando las puntuaciones del individuo son muy diferentes, se habla de incongruencia de estatus. 


\section{Presentación y ANÁlisis De los Resultados}

\subsection{Análisis del empleo general de pero enfático en el habla de Santiago de Chile}

En este estudio hemos encontrado 111 casos en que el pero cumplía una función enfática de un total de 642 ocasiones en que se empleó la partícula, lo que equivale al $17,2 \%$ del total de usos. La mayor cantidad de casos se ubicó en secuencias narrativas, esto es, $76(68,5 \%)$ casos, mientras que en secuencias argumentativas apareció en $35(31,5 \%)$ oportunidades. Como puede apreciarse, el pero enfático es altamente frecuente en el discurso narrativo. A este respecto, una posible explicación se halla en que se trata de narraciones de experiencia personal en el sentido laboviano, donde hay un alto grado de involucramiento con lo narrado (Labov 1997) y, en consecuencia, podría tratarse incluso de un recurso que cumple una función evaluativa dentro de la historia, porque es precisamente un intensificador. Labov (1972) clasifica los elementos evaluativos y señala que los intensificadores son un tipo de recurso evaluativo que permite fortalecer la estructura de la narración. Asimismo, Reilly et al. (1990) ponen de relieve que los intensificadores constituyen marcas que contribuyen a crear fuerza o a enfatizar ciertas acciones que el narrador considera importantes. En este caso, por supuesto, hay que entender la evaluación tal como la definen Labov y Waletzky (1967), esto es, como la sección que es utilizada por el narrador para validar su narración, es decir, para dejar en claro cuál es la razón de ser de su relato y su meta al narrarlo. De este modo, la evaluación marca la parte central o informativa de un relato, aunque puede ser transversal a este. La evaluación no constituye estrictamente una parte, sino que está conformada por todos los fragmentos en que el narrador utiliza medios que hacen de la historia un relato interesante.

En segundo término, hemos encontrado cinco elementos enfatizados con pero, que revisamos y ejemplificamos a continuación.

1. Sustantivos: en esta categoría hemos agrupado los casos en que el pero enfatiza a un sustantivo. Por ejemplo, en (1), caso extraído de una secuencia narrativa, se enfatiza un sustantivo con función de sujeto:

(1) nunca me ha pasado nada / nada tan heavy porque hay personas que me han contado que la han pasado pero miles de cosas y yo / de verdad / de repente como que no creo mucho (SCHI_M12_048).

(2) (...) por aquí yo me acuerdo cuando éramos chicos vivían familias vivían yo tenía pero ene de amigos (SCHI_H23_088).

En el ejemplo (2) se enfatiza el sustantivo con función de complemento directo, y el discurso sigue con su misma dirección. En este caso, la evaluación del hablante requiere de un realce del grupo nominal que le sigue, que supone un énfasis en el tipo de relación con otros.

2. Adjetivos: combinación de pero + adjetivo, por ejemplo:

(3) (...) y esa vez estábamos jugando / porque era de esas casas antiguas / donde hay un patio pero inmenso / no hay antejardín / ¿cachái? pero es inmensa así (...) (SCHI_M12_048). 
(4) la C estaba en el colegio / se suponía que era casi la misma hora que tenía que llegar a la casa / entonces fue como todo como que / concordó todo/ entonces fue pero atroz (SCHI_M21_020).

En ambos casos, como es evidente, se trata de la combinación pero + adjetivo. Esta combinación presenta muy poca frecuencia en el corpus y sólo se halla en narraciones. Los datos específicos serán expuestos con posterioridad.

3. Adverbios: en esta categoría incluimos los adverbios que expresan modo, como los terminados en -mente o en -ísimo. Asimismo, se incluye la construcción pero + gerundio, como muestra el ejemplo que sigue.

(5) (...) ya/ seguimos caminando con el viejito ¿cachái? / y en eso aparece el otro huevón po ¿cachái? el que era él y empezamos a caminar los tres/ y yo ingenua a morir no sabía que me estaba pero metiendo / ya (...) (SCHI_M11_010).

En el ejemplo narrativo (5) presenciamos la construcción pero + gerundio. Esta construcción es frecuente cuando lo que se enfatiza con la partícula pero corresponde a un adverbio.

(6) (...) lamentablemente el vocabulario deja mucho que desear pero mucho mucho (SCHI_H31_025).

Por su parte, en el ejemplo (6), tomado de una secuencia argumentativa, nos encontramos frente a un caso en el que pero enfatiza a un adverbio. Este ejemplo es particularmente interesante, dado que se complementa, a su vez, con la categoría "reduplicaciones", que revisaremos en lo que sigue.

4. Reduplicaciones: se trata de la unión de los miembros realzados. Los elementos reduplicados pueden ser sustantivos, adjetivos o adverbios. Por ejemplo:

(7) (...) no / no percances no más que han sido operado de de cosas así pasajeras no tanto grave / grave grave que tenga que estar no / cosas que le han pasado a las tres pero no tan grave grave así (SCHI_M11_012).

En el ejemplo (7) se reduplica la negación.

(8) eh sí pero yo siempre fui / medio payaso como el como el de la fiesta o sea siempre andaba con el chiste o la broma adelante y me recuerdo que en los malones que hacían ma / antes se llamaban malones o / eeh había copete pero hay copete pero se curan todos (...) (SCHI H21_017).

En el ejemplo precedente, se puede observar que el pero une los miembros realzados: "había copete pero hay copete". Ambos ejemplos fueron tomados de 
secuencias narrativas, ya que en los discursos argumentativos analizados no se observaron reduplicaciones enfatizadas con pero.

5. Oraciones y grupos preposicionales: en esta categoría hemos agrupado los casos que no habían sido descritos por la bibliografía revisada como elementos enfatizados con pero ${ }^{3}$. Básicamente, se incluyen oraciones completas y subordinadas, además de grupos preposicionales. Estos casos suponen distintos sentidos y funciones que se detallan a continuación.

(9) fuimos a ver una viejita / y la viejita cuando ya antes que llegará me dijo “ah ya sé a qué viene usted ¿qué le pasó?" le empecé a contar pues // y ella me trató me trató y fíjate que me que me haya sanado / pero que tenía los tendones hecho pelotitas (...) (SCHI_H31_030).

(10) y yo la saco de huevas abro mi billetera para pasarle mi carnet pues huevón y el huevón me mira así y me dice "aah pero tiene plata / déjeme plata" / y yo huevón le pasé setenta lucas / la pelotuda (SCHI_ M11 010).

(11) y me querían quitar la cartera / y de tanto que grité / grité y grité / que un caballero que venía hacia arriba también / en bicicleta // los asustó a ellos / los gritoneó / y el caballero prácticamente que me salvó porque los chiquillos no insistieron / no me hicieron nada felizmente / pero tampoco me quitaron la cartera (SCHI_M12_044).

(12) en ese momento igual me apoyó mucho pero en ese minuto igual estaba como mal así como mi mamá tenía el único trabajo (SCHI_M11_009).

(13) o sea / no sé / no te sabría decir / ahora obviamente que cuando uno está en una etapa de la vida / la última etapa es la que está viviendo / y por lo tanto uno dice bueno estos son / mis mis amigos// (...) //I: pero sería terriblemente poco objetivo decir que los amigos / de hace años no cierto / no no son buenos amigos o no fueron buenos amigos en su momento (...) (SCHI_H31_030).

En el ejemplo (9), tomado de una narración, la partícula pero está enfatizando a una oración subordinada. En el (10) se observa el énfasis de una oración simple (tiene plata).

En los ejemplos (11) y (12), también extraídos de una narración, se observa más claramente como el pero supone un énfasis a una apreciación o exclamación; en el primer caso el hecho de no ser asaltada a pesar de la situación problemática a la que se enfrenta; en el segundo caso la ayuda oportuna que recibe el sujeto ante su gran malestar

Si bien se trata de una categoría amplia, que presenta, además, la mayor concentración de casos, no hemos querido desagregarla, debido a que se trata de un primer intento por determinar cuáles son los elementos enfatizados con pero en el habla santiaguina. En este caso, en particular, se trata de una primera aproximación a una nueva categoría de análisis no contemplada en la bibliografía revisada. Futuras investigaciones podrían concentrarse en diferenciar las modalidades que expresan las oraciones, recategorizando, a su vez, los grupos preposicionales. 
(enfatizado por el pero). Gramaticalmente, por otro lado, en (11) el énfasis recae en una oración completa, mientras que en (12) lo hace en un complemento circunstancial. Por su parte, en (13), el discurso sigue en la misma dirección; el informante no está contraponiendo una idea, sino que sigue argumentando en el sentido anterior. La partícula pero, en este caso, también está enfatizando la oración completa.

Otro caso, que también supone un énfasis en una oración, refiere a las apelaciones directas, en cuyo caso el elemento enfatizado es un requerimiento directo o llamado a otro oyente:

(14)pero imagínate mis / mis amigos todos los que me conocían así / qué te pasó que caíste tan bajo una casa tan chica / tan / yo le dije por enfermedad me viene en prueba aquí a trabajar y me resultó (SCHI_ H31_030).

(15) nos pusimos a hablar con el otro gallo pues / entonces el viejo va y me dice pero mijita sabe qué me dijo yo prefiero que vaya usted porque yo no tengo confianza en el otro tipo (SCHI_M11_010).

(16) me acuerdo que el huevón me estaba contando cuando íbamos para allá / que había hecho una / iba / echado una con la señora /pero ves ¿cachái? pues / / la cuestión que / el huevón se quedó dormido po / y el huevón me / me dijo que no había / se / que no pasaba nada po (SCHI_H11_001).

Si bien es cierto, el ejemplo (15) pertenece a la categoría sustantivo, es interesante de mencionar pues se relaciona con el tipo de uso del pero expuesto en el ejemplo (14), ya que supone una apelación en cuanto elemento vocativo. En ambos casos, el énfasis recae en un requerimiento de atención o apelación a otro. Esta necesidad se observa, además, asociada a preguntas retóricas, como en el caso (16) en el que no se contraponen dos términos o miembros; todo el elemento podría ser reemplazado por “¿te das cuenta?” con un énfasis o realce asociado.

Los ejemplos siguientes también corresponden a énfasis en oraciones completas, aunque con algunos usos particulares:

(17) bueno al final el tipo se bajó pero eso ha sido lo más relevante que me ha tocado pasar en el T cuando recién empezó (SCHI_M11_008).

(18) y en eso aparece otro gallo así / entonces el gallo va y pregunta el abuelito le pregunta al gallo oiga usted sabe dónde queda tal dirección y el gallo le dice "sí" le dijo "eeh pero es / queda a dos cuadras más allá" un ejemplo "pero y voy al banco ahora y si me desocupo yo los sigo y los acompaño" / ya seguimos caminando con el viejito ¿cachái? (SCHI_M11_010).

(19) puede andar dos tres cuatro ocho diez micro en un tramo de tiempo y te cobran una / pero me encanta porque hay menos micros / hay menos bulla 
en el centro / ehh / no andái con plata en el bolsillo / ehh /los choferes no están estresados / ¡manejan! / es todo lo que hacen (SCHI_M23_094).

(20) a pesar de que no tengo estudios / no tengo experiencia / eh en algo profesional // pero Dios siempre está ahí // siempre está allí / proveyendo de cosas que uno necesita (SCHI_M21_019).

En los ejemplos (17), (18), (19), en los que los dos primeros son de narraciones y el último de una argumentación, se observa un uso enfático asociado a oraciones completas. En ninguno de los ejemplos el valor de la partícula es adversativo. Es posible elidirla en las narraciones expuestas, pues apuntan a la misma orientación discursiva y al mismo sentido. Parece poseer un valor copulativo en tanto puede ser reemplazada por la partícula "y". La posibilidad de elisión, se observa además en otros casos, en los que el "pero" no posee otro valor sino el énfasis, como en el ejemplo (20). Un ejemplo similar, pero asociado más bien a la partícula "entonces" se observa en un caso extraído de las secuencias argumentativas:

(21) claro / entonces yo quedé con la duda y me metí la mano en el bolsillo cuando me bajé y no lo tenía ya / y ahí me lo robó pero me sentí mal porque por primera vez que me pasaba pues (SCHI_M23_094).

Por último, se incluyeron en esta categoría los elementos enfatizados que corresponden a grupos preposicionales que, en su mayoría, cumplían la función de complemento circunstancial.

(22) (...) eso haría / ese es el cambio que me gustaría/ irme lejos pero para el sur (SCHI_H11_004).

En este caso tomado de una argumentación, el énfasis está puesto en un punto espacial específico. Del total "lejos" se escoge una parte identificada como "sur". El énfasis, en tanto, se encuentra en la dirección. Funciona, en consecuencia, como un complemento circunstancial.

Como se puede apreciar en esta presentación de resultados, la categoría oraciones y grupos preposicionales en el español de Chile parece adquirir una particular relevancia, ya que es la que presenta la mayor cantidad de casos en el corpus analizado. Lo interesante es que las investigaciones revisadas no incluyen los elementos que hemos descrito en este trabajo. Exponemos en lo que sigue, los gráficos correspondientes a la distribución de frecuencias de los elementos enfatizados con pero, según el tipo de secuencia discursiva, a fin de contrastar su empleo.

El Gráfico 1 da cuenta de que la mayor cantidad de elementos enfatizados con pero en las secuencias narrativas son los que representan a la categoría oraciones y grupos preposicionales. Se trata de oraciones y otros elementos que adquieren este matiz enfático, como muestran los ejemplos (9) al (22). En concreto, de las 76 ocasiones en las que se emplea la partícula en estudio, $41(53,9 \%)$ corresponden a la categoría oraciones y grupos preposicionales. Le siguen, en orden de frecuencia, los sustantivos y los adverbios, con apenas $10(13,2 \%)$ casos cada uno. Con $8(10,5 \%)$ apariciones se hallan las reduplicaciones y, finalmente, con apenas $7(9,2 \%)$ usos se ubican los adjetivos enfatizados con pero. 
Gráfico 1: Porcentaje de frecuencias de los elementos enfatizados con pero en secuencias narrativas

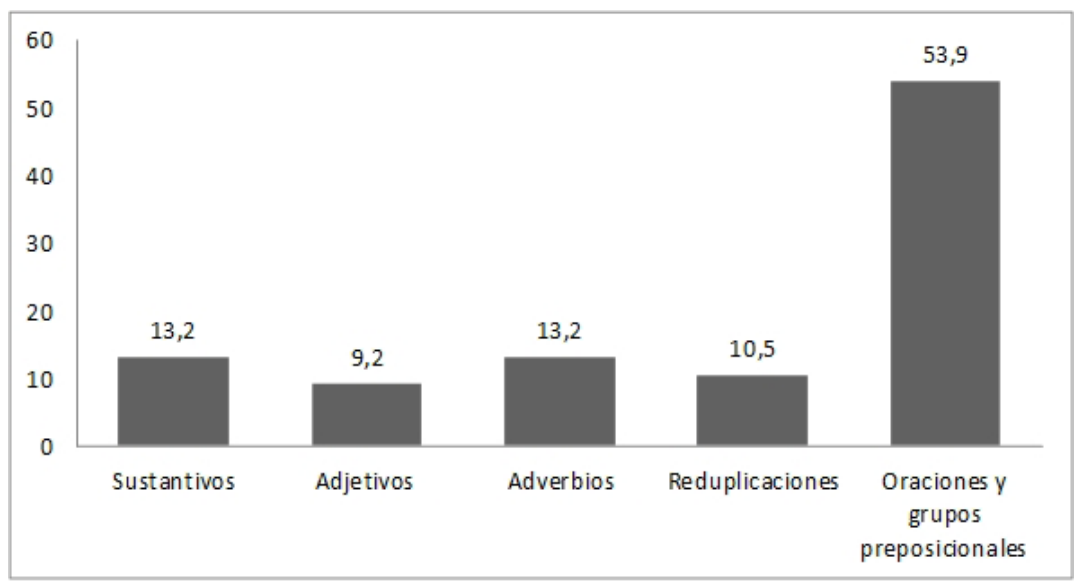

En lo que respecta a los elementos enfatizados con pero en el marco de las secuencias argumentativas, podemos mencionar, tal como se aprecia en el Gráfico 2 , que sólo es utilizado en 3 de 5 elementos. Al igual que en las secuencias narrativas, la mayor parte de los casos se observó en la categoría oraciones y grupos preposicionales, que fue empleada en 17/35 (48,6\%) oportunidades. En 14/35 (40\%) casos se hallan los sustantivos y, por último, en apenas $4 / 35(11,4 \%)$ se ubican los adverbios enfatizados con la partícula pero.

Gráfico 2: Porcentaje de frecuencias de los elementos enfatizados con pero en secuencias argumentativas

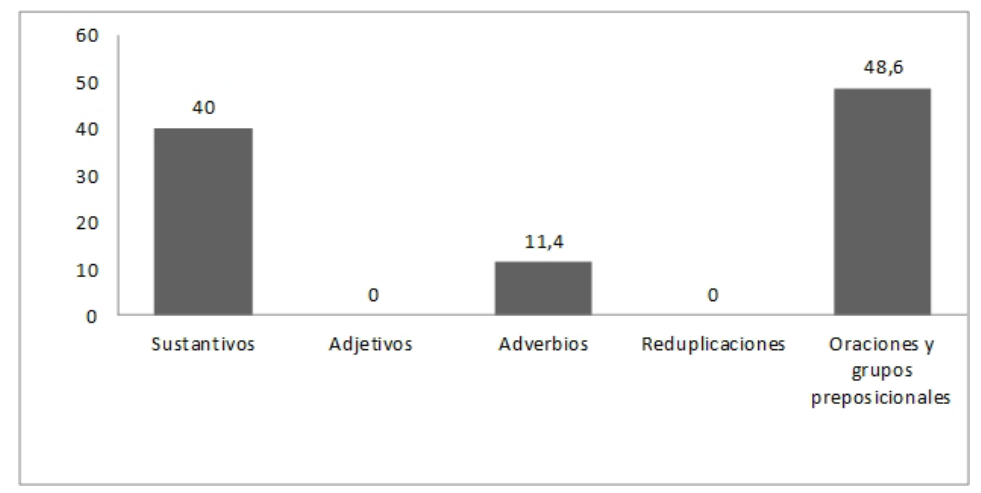

4.2. Análisis del pero enfático en correlación con los factores sociales en el habla de Santiago de Chile

En este apartado revisaremos, en detalle, el empleo de pero enfático en correlación con los factores sociales sexo, edad y nivel educacional de los informantes. En lo que 
respecta al empleo de pero enfático en las estructuras narrativas, este se presentó en 76/111 ocasiones, que se distribuyen porcentualmente, según los factores sociales, como sigue.

Gráfico 3: Porcentaje de frecuencia del empleo de pero enfático según los factores sociales en secuencias narrativas

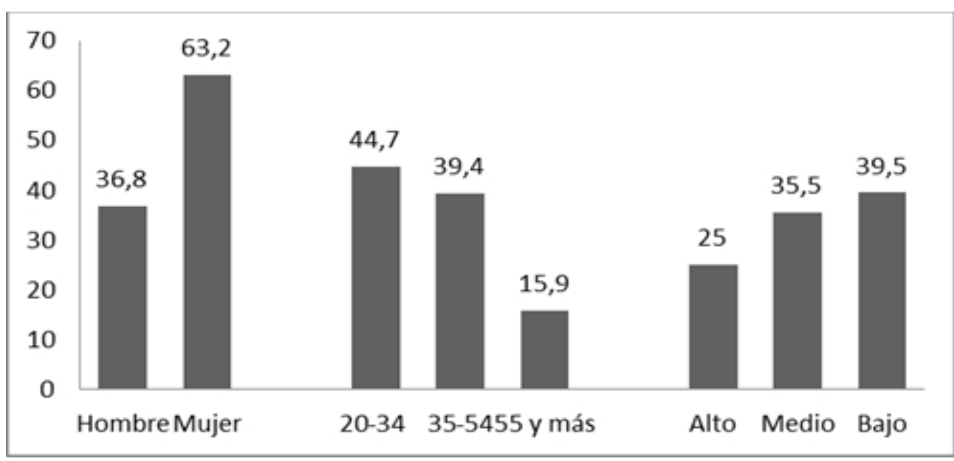

Como se desprende del gráfico precedente, en lo que respecta al factor sexo, son las mujeres quienes emplean más esta partícula en sus relatos, a saber, en 48/76 $(63,2 \%)$ oportunidades, mientras que los hombres lo hacen en $28 / 76(36,8 \%)$ casos.

En cuanto al factor edad, son los hablantes jóvenes quienes más utilizan el pero enfático, en 34/76 (44,7\%) casos. Le siguen los hablantes de 35 a 54 años, en 30/76 $(39,4 \%)$ ocasiones y, finalmente, se ubican los hablantes de 55 años y más, en 12/76 $(15,9 \%)$ oportunidades.

Por último, en relación con el factor nivel educacional, la mayor cantidad de usos se registra en los hablantes del grupo bajo, con 18/76 (39,5\%). Se ubican, luego, los del grupo medio, en 30/76 (35,5\%) casos y, finalmente, los del grupo alto en 12/76 $(25 \%)$ ocurrencias totales.

Para complementar este análisis, hemos realizado pruebas estadísticas. La prueba de varianza no arrojó datos significativos, por lo que hicimos una prueba de ji cuadrado por ausencia y presencia de empleo del pero enfático, cuyo resultado fue estadísticamente significativo para el factor edad, donde $p=0,030$. Los datos de la tabla de contingencia respectiva son los que muestra el Gráfico 4.

La lectura del gráfico precedente nos indica que tal como sugería el Gráfico 3, es el factor edad el que muestra significación, por consiguiente, podríamos pensar que en el grupo de mayor edad disminuye el empleo de pero con función enfática y que esta podría ser una característica de la comunidad de habla analizada.

Debido a la escasa presencia del fenómeno en estudio, presentamos, en la Tabla 2, su distribución detallada según el tipo de elemento enfatizado.

Como se puede apreciar en la Tabla 2, de los 76 casos en los que la partícula pero funcionó con valor enfático en las narraciones, $41 / 76(53,9 \%)$ corresponden a la categoría oraciones y grupos preposicionales, $10 / 76(13,1 \%)$ a la categoría sustantivos y adverbios, 8/76 (10,5\%) a la categoría reduplicaciones, y 7/76 (9,2\%) a la categoría adjetivos. 
Gráfico 4: Presencia y ausencia de pero enfático según el factor edad

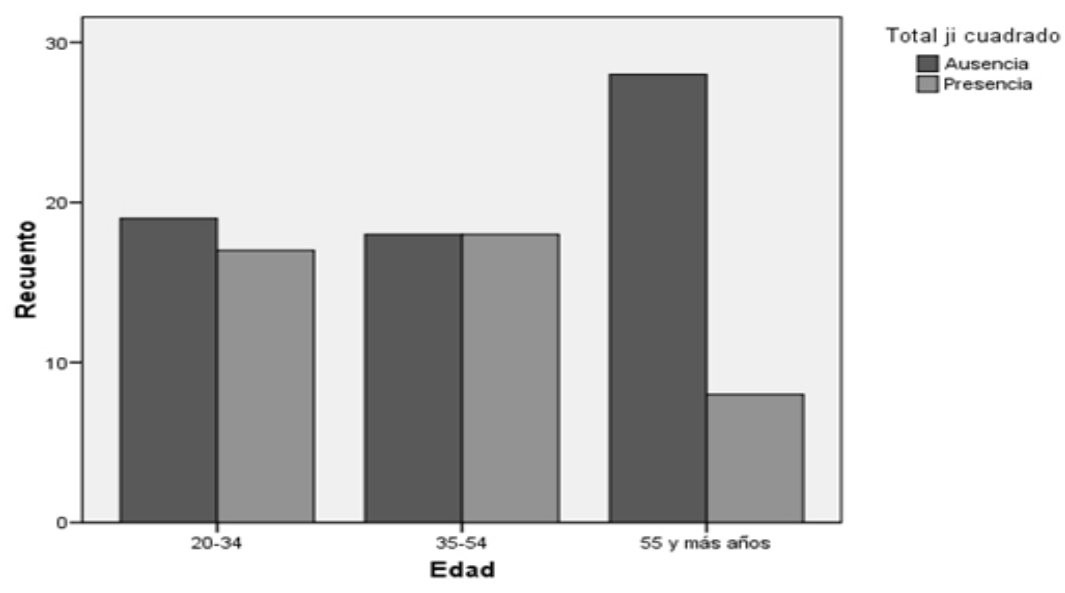

Tabla 2: Distribución de frecuencia de los elementos enfatizados con pero en secuencias narrativas

\begin{tabular}{|l|l|l|l|l|l|l|}
\hline Variable & Variable & $\begin{array}{l}\text { Oraciones y } \\
\text { grupos } \\
\text { prepisicionales }\end{array}$ & Sustantivo & Adverbios & Reduplicaciones & Adjetivos \\
\hline \multirow{2}{*}{ Sexo } & Hombre & $48,8(20 / 41)$ & $20(2 / 10)$ & $20(2 / 10)$ & $75(6 / 8)$ & $57,1(4 / 7)$ \\
\cline { 2 - 7 } & Mujer & $51,2(21 / 41)$ & $80(8 / 10)$ & $80(8 / 10)$ & $25(2 / 8)$ & $42,9(3 / 7)$ \\
\hline \multirow{2}{*}{ Edad } & $20-34$ años & $34,1(14 / 41)$ & $80(8 / 10)$ & $60(6 / 10)$ & $37,5(3 / 8)$ & $42,8(3 / 7)$ \\
\cline { 2 - 7 } & $35-54$ años & $51,2(21 / 41$ & $10(1 / 10)$ & $20(2 / 10)$ & $50(4 / 8)$ & $57,1(4 / 7)$ \\
\cline { 2 - 7 } & 55 años y más & $14,7(6 / 41)$ & $10(1 / 10)$ & $20(2 / 10)$ & $12,5(1 / 8)$ & $0(0 / 7)$ \\
\hline \multirow{2}{*}{$\begin{array}{l}\text { Nivel } \\
\text { educacional }\end{array}$} & Alto & $12,2(5 / 41)$ & $40(4 / 10)$ & $40(4 / 10)$ & $25(2 / 8)$ & $57,1(4 / 7)$ \\
\cline { 2 - 7 } & Medio & $43,9(18 / 41)$ & $30(3 / 10)$ & $10(1 / 10)$ & $25(2 / 8)$ & $14,3(1 / 7)$ \\
\cline { 2 - 7 } & Bajo & $43,9(18 / 41)$ & $30(3 / 10)$ & $50(5 / 10)$ & $50(4 / 8)$ & $28,6(2 / 7)$ \\
\hline
\end{tabular}

En relación con el factor sexo, excepto en las reduplicaciones y adjetivos, son las mujeres las que utilizan el pero enfático con mayor frecuencia que los hombres. Es relevante que este rasgo aparezca como una marca en las narrativas femeninas, que son las que suelen mostrar la mayor cantidad de recursos de intensificación (cf. Guerrero 2014). De esta manera, insistimos en que la partícula en estudio permite reforzar la idea, adquiriendo, por lo tanto, un sentido aumentativo, que es el que se ajusta al sentido de énfasis propiamente.

En lo que respecta al factor edad, tanto los sustantivos como los adverbios son enfatizados con pero sobre todo por el grupo de 20 a 34 años, mientras que las categoría oraciones y grupos preposicionales, adjetivos y reduplicaciones son más enfatizadas por los hablantes del grupo de edad intermedia (35 a 54 años). En general, son los sujetos del grupo de 55 años y más los que menos emplean el pero enfático con valor enfático. 
Finalmente, en relación con el nivel educacional, las categorías oraciones y grupos preposicionales y los sustantivos suelen ser recurrentes en los hablantes de los grupos medio y bajo, y las categorías adverbios, reduplicaciones son más frecuentes sólo en los hablantes del grupo bajo. Sólo en la categoría adjetivos, son los hablantes del grupo alto los que destacan en frecuencia.

En otro orden, en el marco de las estructuras argumentativas, el pero enfático se utilizó apenas en 35/111 oportunidades. Dichos casos se distribuyen porcentualmente, como muestra el siguiente gráfico de factores sociales:

Gráfico 5: Porcentaje de frecuencia del empleo de pero enfático según los factores sociales en secuencia argumentativa

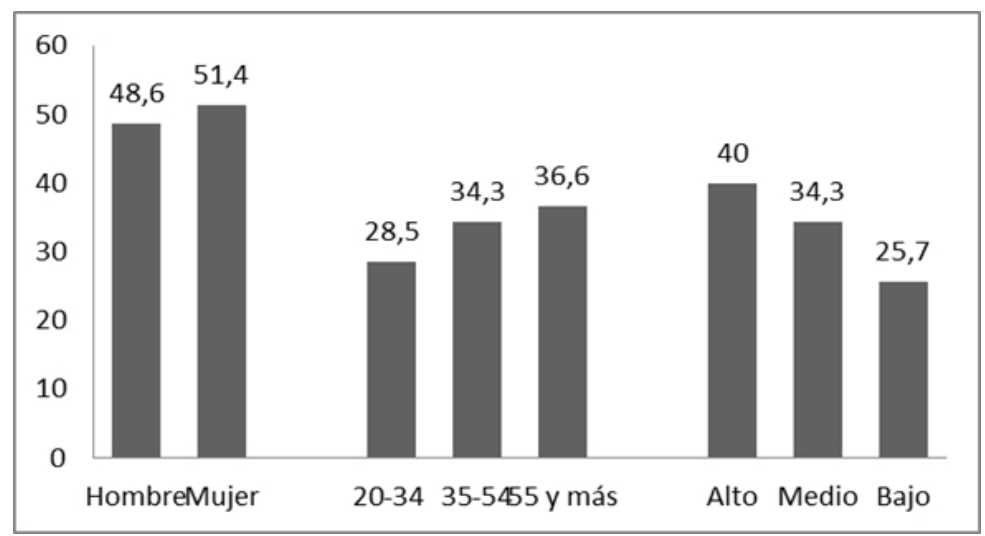

Como se puede apreciar en el Gráfico 5, en relación con el factor sexo, al igual que en las secuencias narrativas, son las mujeres quienes utilizan con mayor frecuencia el pero enfático, a saber, en 18/35 (51,4\%) ocasiones. Los hombres emplean esta partícula en $17 / 35(48,6 \%)$ oportunidades. No obstante, se trata de diferencias porcentuales mínimas.

En lo que dice relación con el factor edad, se produce una curva inversa respecto de la utilización de pero en las narraciones, esto es, son los hablantes de 55 años y más quienes más lo emplean, en 13/35 (36,6\%) casos. Se ubican, a continuación, los informantes del grupo de edad intermedia con 12/35 (34,3\%) casos y, por último, los del grupo joven con 10/35 (28,5\%) usos totales.

En tercer lugar, en cuanto al nivel educacional también se produce una curva contraria a la percibida en las secuencias narrativas, es decir, son los hablantes del nivel educacional alto los que más prefieren el valor enfático del pero, con 14/35 $(40 \%)$ casos. Los hablantes del grupo medio lo utilizaron en 12/35 (34,3\%) ocasiones y los informantes del grupo bajo en $9 / 35(25,7 \%)$ oportunidades del total de la muestra.

Este análisis, además, se complementó con un análisis de varianza, que arrojó que la prueba de los efectos intersujetos de las variables externas edad y nivel educacional es estadísticamente significativa $(\mathrm{p}=0,045)$. El gráfico que muestra este hallazgo es el que sigue: 
Gráfico 6: Análisis de varianza del empleo de pero enfático y la interacción de los factores edad y nivel educacional en secuencias argumentativas

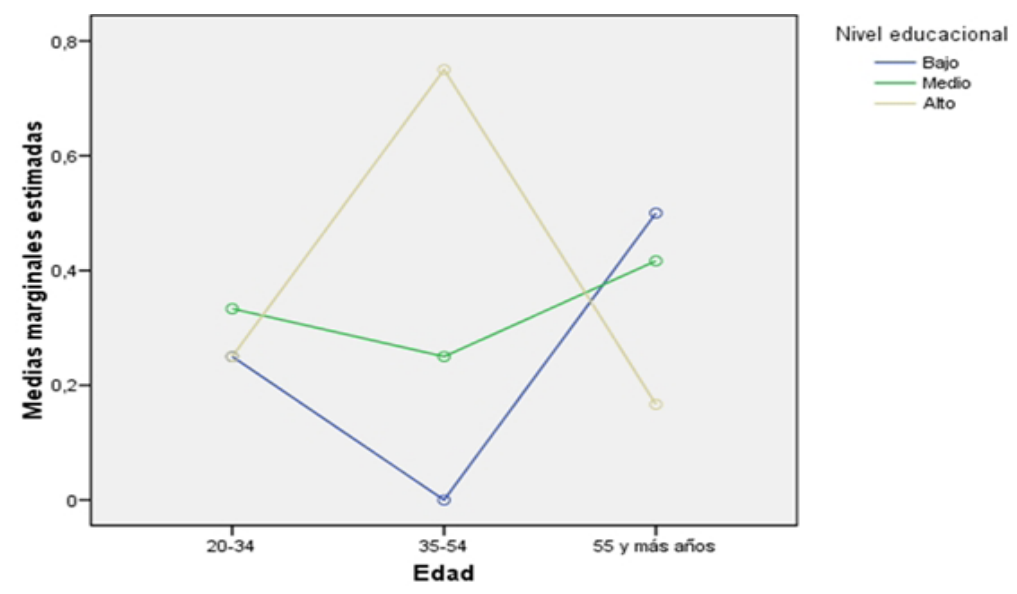

El Gráfico 6, como puede apreciarse, muestra un comportamiento disordinal entre las variables en estudio. Es decir, el grupo educacional Alto se comporta de manera inversa a los grupos Bajo y Medio. Este comportamiento inverso se acentúa en el grupo de edad intermedia. Estos datos coinciden con el gráfico 5 de porcentajes, que también muestra este comportamiento inverso en las variables edad y nivel educacional.

Por tratarse de una frecuencia muy menor del empleo del pero con valor enfático, presentamos, en la Tabla 3, su distribución detallada según el tipo de elemento enfatizado. En este caso, a diferencia de lo ocurrido en las secuencias narrativas, la partícula en estudio sólo apareció enfatizando tres tipos de elementos, a saber, oraciones y grupos preposicionales, sustantivos y adverbios.

Tabla 3: Distribución de frecuencia de los elementos enfatizados con pero en secuencias argumentativas

\begin{tabular}{|l|l|l|l|l|}
\hline Variable & Variable & $\begin{array}{l}\text { Oraciones y } \\
\text { grupos } \\
\text { prepisicionales }\end{array}$ & Sustantivo & Adverbios \\
\hline \multirow{3}{*}{ Sexo } & Hombre & $41,2 \%(7 / 17)$ & $57,1 \%(8 / 14)$ & $50 \%(2 / 4)$ \\
\cline { 2 - 5 } & Mujer & $58,8 \%(10 / 17)$ & $42,9 \%(6 / 14)$ & $50 \%(2 / 4)$ \\
\hline \multirow{3}{*}{ Edad } & $20-34$ años & $23,5 \%(4 / 17)$ & $35,7 \%(5 / 14)$ & $25 \%(1 / 4)$ \\
\cline { 2 - 5 } & $35-54$ años & $29,5 \%(5 / 17)$ & $42,9 \%(6 / 14)$ & $25 \%(1 / 4)$ \\
\cline { 2 - 5 } & 55 años y más & $47 \%(8 / 17)$ & $21,4 \%(3 / 14)$ & $50 \%(2 / 4)$ \\
\hline \multirow{2}{*}{$\begin{array}{l}\text { Nivel } \\
\text { educacional }\end{array}$} & Alto & $35,3 \%(6 / 17)$ & $50 \%(7 / 14)$ & $25 \%(1 / 4)$ \\
\cline { 2 - 5 } & Medio & $29,4 \%(5 / 17)$ & $42,8 \%(6 / 14)$ & $25 \%(1 / 4)$ \\
\cline { 2 - 5 } & Bajo & $35,3 \%(6 / 17)$ & $7,2 \%(1 / 14)$ & $50 \%(2 / 4)$ \\
\hline
\end{tabular}


Como se desprende de los datos expuestos en la Tabla 3, de los 35 casos en los que la partícula pero funcionó con valor enfático en las secuencias argumentativas analizadas en este estudio, $17 / 35(48,6 \%)$ corresponden a la categoría oraciones y grupos preposicionales, $14 / 35(40 \%)$ a la categoría sustantivos y $4 / 35(11,4 \%)$ a la categoría adverbios. En las secuencias argumentativas no se registraron ni adjetivos ni reduplicaciones enfatizadas con pero.

En relación con el factor sexo, son las mujeres las que utilizan el pero enfático con mayor frecuencia que los hombres; en el caso de los adverbios la frecuencia es idéntica y se trata de una cantidad mínima de casos.

En lo que respecta al factor edad, tanto la categoría oraciones y grupos preposicionales como los adverbios son enfatizados con pero, mayormente por el grupo de 55 años y más; mientras que la categoría sustantivos es más enfatizada por los hablantes del grupo de edad intermedia (35 a 54 años).

En tercer lugar, en cuanto al nivel educacional, son los hablantes de los grupos alto y bajo los que prefieren la categoría oraciones y grupos preposicionales. Los sustantivos enfatizados con pero son preferidos por los hablantes de los grupos alto y medio, y por último, los hablantes del grupo bajo suelen enfatizar adverbios más que los hablantes de los grupos alto y medio. Esta última categoría presenta una frecuencia de aparición mínima en la muestra analizada.

\section{CONCLUSIONES}

En esta investigación hemos analizado el comportamiento del pero enfático a nivel discursivo y la estratificación sociolingüística del empleo de dicho mecanismo en el corpus PRESEEA de Santiago de Chile. Las conclusiones más relevantes de nuestra indagación pueden sintetizarse del siguiente modo:

1. En relación con el objetivo que buscaba determinar cuáles son los elementos enfatizados con pero, hemos concluido que son sustantivos, adjetivos, adverbios, reduplicaciones y otros que no habían sido descritos en la bibliografía revisada, esto es, oraciones y grupos preposicionales. Además, se consideran en esta categoría otros valores, como el énfasis a las apelaciones directas. También hemos encontrado un cuarto sentido a esta partícula, el de requerimiento o necesidad de información.

2. Asimismo, concluimos que el elemento en estudio es mayormente empleado en discursos narrativos, que se caracterizan por su alto grado de involucramiento. De hecho, sólo en los relatos analizados aparecieron los cinco elementos enfatizados con pero, mientras que en las secuencias argumentativas sólo se presentaron tres de ellos. Sobre la frecuencia de empleo de dicha partícula hemos comprobado que, en las secuencias narrativas, la mayor frecuencia del pero enfático se ubica en la categoría oraciones y grupos preposicionales (41/76). El resto de los casos se distribuyen entre los sustantivos y los adverbios (19/76 en cada categoría), las reduplicaciones (8/76) y los adjetivos (7/76). En lo que respecta a las secuencias argumentativas, también es la categoría oraciones y grupos preposicionales la que más se destaca (17/35), aunque los sustantivos 
también son enfatizados con pero de manera recurrente (14/35). Con una frecuencia menor se ubican los adverbios (4/35).

3. En otro orden, pudimos comprobar parcialmente nuestra hipótesis intragrupal, esto es, que se trata de un mecanismo empleado mayormente por mujeres, especialmente, en secuencias narrativas. Sin embargo, no pudimos comprobar nuestra hipótesis inter-grupal, aunque el factor edad mostró significación estadística en el marco de las narraciones y, por lo tanto, concluimos que efectivamente se trata de un recurso más propio del habla joven de la comunidad de habla en estudio.

4. Finalmente, destacamos la relevancia de considerar el pero enfático como un fenómeno variable desde la perspectiva sociolingüística. No obstante, parece oportuno destacar el carácter limitado de los hallazgos y de las interpretaciones de la investigación aquí expuesta. Futuros trabajos respecto del fenómeno estudiado posibilitarán verificar la validez de estos hallazgos. Asimismo, permitirán desagregar la nueva categoría propuesta en este estudio.

\section{OBRAS CITADAS}

Acín, Esperanza. 1993-1994. "Sobre pero enfático". Cuadernos de investigación filológica 19-20: 219-233.

Alarcos Llorach, Emilio. 1999. Gramática de la lengua española. Madrid: Espasa Calpe.

Blas Arroyo, José Luis. 2005. Sociolingüística del español. Desarrollos y perspectivas en el estudio de la lengua española en contexto social. Madrid: Cátedra.

Coates, Jennifer. 2009. Mujeres, hombres y lenguaje. Un acercamiento sociolingüistico a las diferencias de género. México: Fondo de Cultura Económica.

Gili Gaya, Samuel. 1961. Curso superior de sintaxis española. Barcelona: Spes.

Guerrero, Silvana. 2014. Variación discursiva en narraciones de experiencia personal en el español hablado en Santiago de Chile. Tesis para optar al grado de Doctora en Lingüística. Chile: Pontificia Universidad Católica de Chile.

Hernández Campoy, Juan Manuel y Manuel Almeida. 2005. Metodología de la investigación sociolingüistica. Málaga: Editorial Comares.

Labov, William. 1972. "The transformation of experience in narrative syntax". En Language in the inner city. Studies in the black English vernacular. Filadelfia, Estados Unidos: University of Pennsylvania Press: 354-375.

Labov, William. 1983 [1972]. Modelos sociolingüisticos. Madrid: Cátedra.

Labov, William. 1997. Some further steps in narrative analysis. Journal of narrative and life history 7: 395- 415.

Labov, William y Josua Waletzky. 1967. "Narrative analysis". En Helm, Jane (ed.), Essays on the verbal and visual arts. Seattle: University of Washington Press: 12-44.

Lenski, Gerhardt. 1954. Status Crystallization: A Non-vertical Dimension of Social Status. American Sociological Review, 19, 405-413.

López Morales, Humberto. 1994. Métodos de investigación lingüistica. Salamanca: Ediciones Colegio de España.

Moreno Fernández, Francisco. 1990. Metodología sociolingüística. Madrid: Gredos.

Moreno Fernández, Francisco. 1998. Principios de sociolingüistica y sociología del lenguaje. Barcelona: Ariel. 
Moreno Fernández, Francisco. 2012. Sociolingüistica cognitiva. Proposiciones, escolios y debates. Madrid/Frankfurt: Iberoamericana/Vervuert.

Nicolás Catabella, Elena y José Antonio Hernández. 2011. Aproximación a la partícula pero desde una perspectiva integradora. Revista Philologica Romanica. Vol. 11: 1010-121.

Prieto, Luis. 1995-1996. Análisis sociolingüístico del dequeísmo en el habla de Santiago de Chile. Boletín de Filología Tomo XXXV: $379-452$.

Real Academia Española. 2001. Diccionario de la lengua española (22. ${ }^{a}$ ed.). Madrid, España: Autor.

Real Academia Española. 2009. Nueva gramática de la lengua española. "La conjunción. Sus grupos sintácticos. Las construcciones coordinadas". Madrid: Espasa Libros.

Real Academia Española. 2010. Nueva gramática de la lengua española. Manual. Madrid: Espasa Libros.

Reilly, Judy, Klima, Edwards y Ursula Bellugi. 1990. One more with feeling: affect and language in atypical populations. Development and Psychopathology 2: 367-391.

San Martín, Abelardo y Silvana Guerrero. 2015. Estudio sociolingüístico del español de Chile (ESECH): recogida y estratificación del corpus de Santiago. Boletín de Filología L (1): 221-247.

Serrano, María José. 2011. Sociolingüistica. Barcelona: Ediciones del Serbal.

van Dijk, Teun. 1996. Estructuras y funciones del discurso. Una introducción interdisciplinaria a la lingüística del texto y a los estudios del discurso. México: Siglo XXI Editores. 
